\title{
Análise de velocidade de vento usando cadeia de Markov
}

\author{
Wind speed analysis using Markov chain \\ Análisis de la velocidad del viento utilizando la cadena de Markov
}

José Edvaldo de Oliveira Nunes ORCID: https://orcid.org/0000-0003-4417-1875 Universidade Federal Rural de Pernambuco, Brasil

E-mail: edvaldooliveiranunes@outlook.com

João Valério de Souza Neto

ORCID: https://orcid.org/0000-0002-6861-5517

Universidade Federal Rural de Pernambuco, Brasil

E-mail: jovasone.ufrpe@gmail.com

Maria Marciele de Lima Silva

ORCID: https://orcid.org/0000-0001-5185-2711

Universidade Federal Rural de Pernambuco, Brasil

E-mail: marciele.liima@gmail.com

Natália Moraes Cordeiro

ORCID: https://orcid.org/0000-0001-5294-1353

Universidade Federal Rural de Pernambuco, Brasil

E-mail: nataliamcorddeiro@gmail.com

Ruben Vivaldi Silva Pessoa

ORCID: https://orcid.org/0000-0003-1469-5918

Universidade Federal Rural de Pernambuco, Brasil

E-mail: ruben.vspessoa@ufpe.br

Ikaro Daniel de Carvalho Barreto

ORCID: https://orcid.org/0000-0001-7253-806X

Universidade Federal Rural de Pernambuco, Brasil

E-mail: daniel.carvalho.ib@gmail.com

Lucian Bogdan Bejan

ORCID: https://orcid.org/0000-0002-3292-6065

Universidade Federal Rural de Pernambuco, Brasil

E-mail: lucianbb@gmail.com

Tatijana Stosic

ORCID: https://orcid.org/0000-0002-5691-945X

Universidade Federal Rural de Pernambuco, Brasil

E-mail: tastosic@gmail.com

\begin{abstract}
Resumo
A busca por energias renováveis vem aumentando nas últimas décadas devido, principalmente, às preocupações ambientais como também à crescente demanda energética. A energia eólica é uma das fontes de energia renovável amplamente adotada pelo mundo, sendo a região nordeste do Brasil destacada pela sua grande capacidade de produção. A implementação de parques eólicos carece de estudos preliminares sobre modelagem estatística de velocidade do vento e, entre os métodos propostos, a Cadeia de Markov se mostrou eficiente. Este trabalho apresenta uma análise estatística da velocidade do vento na cidade de Petrolina - PE, com objetivo de aplicar cadeias de Markov de primeira ordem como método probabilístico na modelagem da velocidade do vento a cada hora. Os dados foram obtidos no site do Instituto Nacional de Meteorologia (INMET), correspondendo ao período de 2010 a 2020. Foram utilizadas cadeias de Markov de primeira ordem com dois estados para encontrar as probabilidades de transição, e a variação anual dessas probabilidades e a Entropia normalizada foram analisadas. Os principais resultados mostraram que o modelo proposto se ajusta bem às variações da velocidade do vento em meses específicos do ano e que modelos com mais estados podem ser propostos com o objetivo de diminuir a perda de informação calculada pelo valor da entropia.
\end{abstract}

Palavras-chave: Petrolina; Energia eólica; Modelagem; Entropia.

\begin{abstract}
The search for renewable energy has increased in recent decades, mainly due to environmental concerns as well as the growing energy demand. Wind energy is one of the sources of renewable energy widely adopted in the world, with the northeast region of Brazil highlighted by its large production capacity. The implementation of wind farms lacks preliminary studies on statistical modeling of wind speed and, among the proposed methods, the Markov Chain is known to be efficient. This work presents a statistical analysis of the wind speed in the city of Petrolina - PE, with the objective to apply a first-order Markov chain, a probabilistic method, in the hourly wind speed modeling. Data were obtained from the website of the National Institute of Meteorology (INMET), corresponding to the period 2010 to 2020 . First-
\end{abstract}


order Markov chain with two states was used to find transition probabilities, and anual variation of these probabilities and normalized Entropy was analyzed. The results showed that the proposed model adjusts well to wind speed variations in specific months of the year and that models with more states can be proposed to reduce the loss of information calculated by the entropy value.

Keywords: Petrolina; Wind energy; Modeling; Entropy.

\section{Resumen}

La búsqueda de energías renovables se ha incrementado en las últimas décadas, principalmente debido a preocupaciones ambientales, así como a la creciente demanda de energía. La energía eólica es una de las fuentes de energía renovable ampliamente adoptada en el mundo, destacando la región noreste de Brasil por su gran capacidad de producción. La implementación de parques eólicos carece de estudios preliminares sobre modelado estadístico de la velocidad del viento y, entre los métodos propuestos, se sabe que la Cadena de Markov es eficiente. Este trabajo presenta un análisis estadístico de la velocidad del viento en la ciudad de Petrolina - PE, con el objetivo de aplicar una cadena de Markov de primer orden, un método probabilístico, en la modelación horaria de la velocidad del viento. Los datos se obtuvieron de la página web del Instituto Nacional de Meteorología (INMET), correspondiente al período 2010 a 2020. Se utilizó cadena de Markov de primer orden con dos estados para encontrar probabilidades de transición, y se analizó la variación anual de estas probabilidades y la entropía normalizada. Los resultados mostraron que el modelo propuesto se ajusta bien a las variaciones de velocidad del viento en meses específicos del año y que se pueden proponer modelos con más estados para reducir la pérdida de información calculada por el valor de entropía.

Palabras clave: Petrolina; Energía eólica; Modelado; Entropía.

\section{Introdução}

A escassez de energia tornou-se um problema global devido à expansão da escala de produção e da demanda do consumidor (Bagal, Soltanabad, Dadjuo, Wakil, \& Ghadimi, 2018), bem como as preocupações ambientais que se tornaram um dos principais motivos para a busca de alternativas mais limpas para geração de energia em diversos países, incluindo o Brasil (Kaygusuz, 2012; Simas, \& Pacca, 2013; Sadorsky, 2021). A indústria de energia eólica é considerada uma das mais promissoras para resolver a crise energética enfrentada pelo mundo, tendo em vista que gera pouca poluição e tem baixo custo de produção (Jiao, Huang, Ma, Han, \& Tian, 2018). Além disso, é uma indústria que se desenvolve de forma acelerada, devido, principalmente, a capacidade eólica mundial que cresceu exponencialmente nas últimas décadas (Chitsaz, Amjady, \& Zareipour, 2015), tornando-se uma tecnologia totalmente comercial e orientada para o mercado, competindo com sucesso com o combustível fóssil e a energia nuclear (Van Kooten, Duan, \& Lynch, 2016).

As novas instalações no mercado de energia eólica onshore atingiram $86,9 \mathrm{GW}$, enquanto o mercado de energia eólica offshore atingiu $6,1 \mathrm{GW}$, tornando 2020 o segundo ano mais alto da história para novas instalações eólicas, tanto onshore quanto offshore. Os cinco principais mercados mundiais em 2020 para novas instalações foram China, Estados Unidos, Brasil, Holanda e Alemanha, que combinados representaram $80,6 \%$ das instalações globais no ano passado, e coletivamente mais de $10 \%$ a mais do que em 2019 (GWEC, 2021).

O Brasil é o sexto país de maior produção, com um total de $15,45 \mathrm{GW}$ ao final de 2019 , representando $2,5 \%$ de participação no mercado mundial de capacidade onshore (GWEC, 2019), sendo que seu maior potencial eólico se encontra no Nordeste ( $\sim 18 \%$ do território brasileiro), que produz $85 \%$ da energia eólica (ABEEólica, 2018), região em que está localizada grande parte das usinas instaladas no País (Ruffato-Ferreira, Barreto, Júnior, Silva, Viana, Nascimento, \& Freitas, 2017).

A implementação de parques eólicos requer uma série de estudos preliminares para estimar as características da velocidade do vento e do potencial da energia eólica. Para descrever as características estatísticas da velocidade do vento em um determinado local, o método usual é ajustar os dados experimentais do vento com distribuições de probabilidade (Carta, Ramirez, \& Velazquez, 2009; Jung, \& Schindler, 2019). Outra forma de estimar as características estatísticas do vento consiste em utilizar processos autorregressivos ou cadeias de Markov (Poggi, Muselli, Notton, Cristofari, \& Louche, 2003; Sarmiento, Valencia, \& Akhavan-Tabatabaei, 2018; Shamshad, Bawadi, Hussin, Majid, \& Sanusi, 2005; Xie, Liao, Tai, \& Hu, 2017). Esse tipo de modelagem tem a vantagem de levar em conta as variações temporais da velocidade e direção do vento (Ettoumi, Sauvageot, \& 
Adane, 2003; Masseran, 2015).

O presente trabalho analisa a série temporal da velocidade do vento medida a 10 metros de altura em uma estação da cidade de Petrolina, município localizado na divisa dos estados da Bahia (BA) e Pernambuco (PE), região nordeste do Brasil, considerada uma das mais promissoras para a produção de energia eólica no País (De Figuerêdo, Moreira, Stosic, \& Stosic, 2014). Portanto, neste artigo, objetiva-se aplicar a cadeia de Markov de primeira ordem como método probabilístico na modelagem de medições de velocidade do vento por hora em uma estação de Petrolina - PE.

\section{Metodologia}

A metodologia utilizada para estudar o comportamento da série temporal de velocidade do vento tem como base a análise qualitativa dos dados, a partir de modelos de cadeia de Markov (Balzter, 2000), conforme procedimentos apresentados por Ettoumi, Sauvageot e Adane (2003). A cadeia de Markov de primeira ordem foi aplicada para cada mês do ano (janeiro, fevereiro, ..., dezembro) utilizando dois estados separados por velocidade, que para maioria das turbinas eólicas representa velocidade mínima ("cut in") quando a turbina começa gerar eletricidade: $V<3,5 \mathrm{~m} / \mathrm{s}$ e $V \geq 3,5 \mathrm{~m} / \mathrm{s}$. As matrizes de transição e as probabilidades limite foram calculadas utilizando pacotes disponíveis no software R: markovchain (Spedicato, 2017) e ggplot2 (Wickham, 2016).

\subsection{Descrição dos dados}

A estação meteorológica de Petrolina está localizada nas coordenadas geográficas de longitude $40^{\circ} 30^{\prime} 28^{\prime \prime} \mathrm{W}$; latitude 09 $23^{\prime} 34^{\prime \prime} \mathrm{S}$ e 370,46 m de altitude (Figura 1). Os dados horários de velocidade do vento (em m/s) registrados na altura de 10 metros durante o período 01/01/2010-31/07/2020 foram obtidos no site do Instituto Nacional de Meteorologia (INMET, 2021). 
Figura 1. Localização do município de Petrolina - PE.

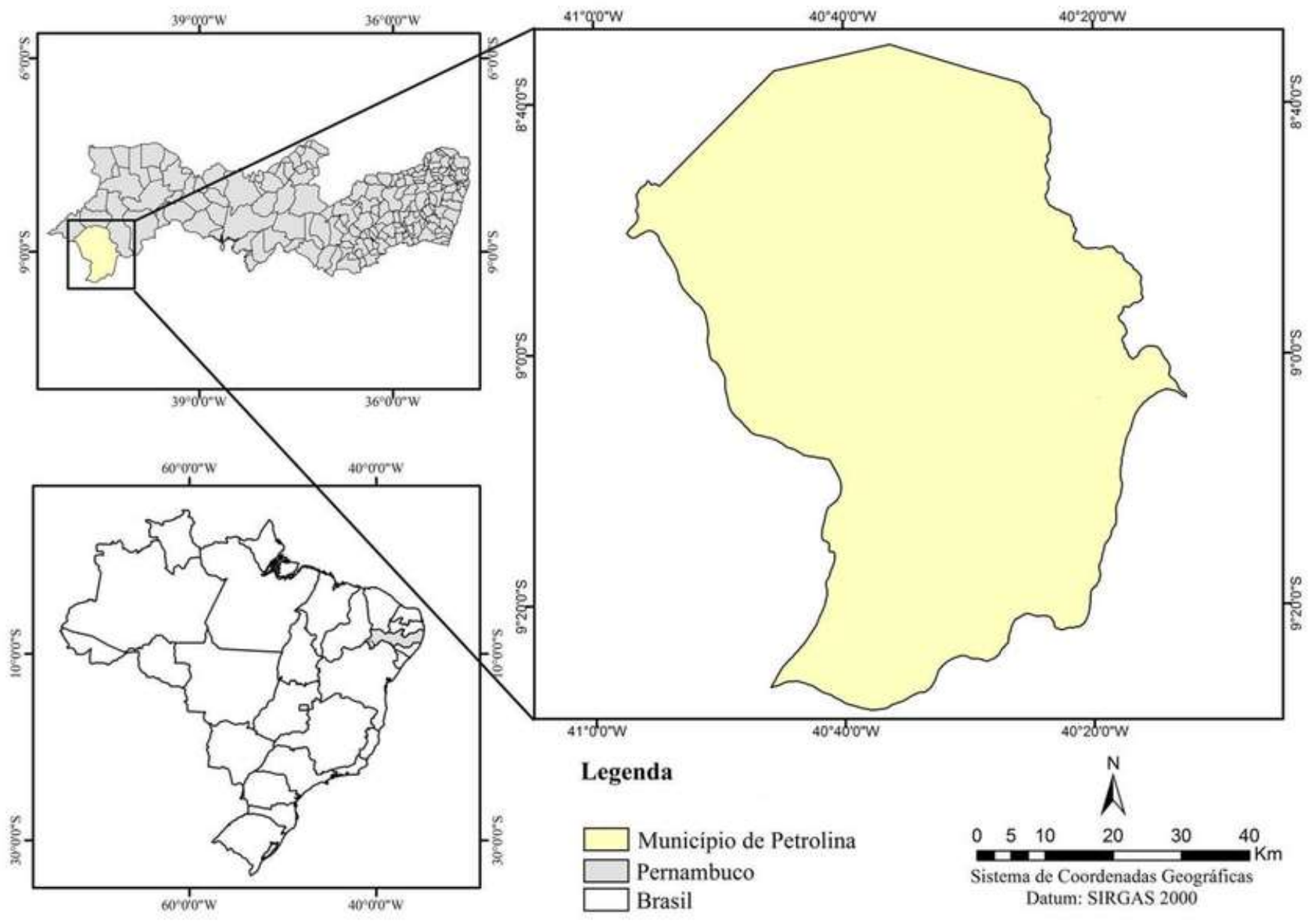

Fonte: Autores (2021).

\subsection{Cadeia de Markov}

Modelos de cadeia de Markov são processos estocásticos que podem ser parametrizados estimando a probabilidade de transição entre estados discretos em um determinado sistema (Balzter, 2000). Na cadeia de Markov de primeira ordem, assumese que a probabilidade de um estado depende apenas do estado anterior.

Seja $X(t)$ um processo estocástico com o espaço de estados discreto $S=\{1,2, \ldots, n\}$, e o intervalo de tempo discreto. A probabilidade de transição de um estado $i$ para um estado $j$ é definida como

$$
P_{i j}=p\{X(t)=j \mid X(t-1)=i\} \forall i, j \in\{1, \ldots, n\} .
$$

Essas probabilidades de transição formam os elementos da matriz de transição $P$. Para $n$ estados, a matriz de transição de primeira ordem é uma matriz $n \times n$,

$$
\left(\begin{array}{cccc}
\mathrm{P}_{11} & \mathrm{P}_{12} & \cdots & \mathrm{P}_{1 \mathrm{n}} \\
\mathrm{P}_{21} & \mathrm{P}_{22} & \cdots & \mathrm{P}_{2 \mathrm{n}} \\
\vdots & \vdots & \ddots & \vdots \\
\mathrm{P}_{\mathrm{n} 1} & \mathrm{P}_{\mathrm{n} 2} & \cdots & \mathrm{P}_{\mathrm{nn}}
\end{array}\right)
$$

Por definição, qualquer probabilidade de estado varia entre zero e um,

$$
0<P_{i j}<1 \text {. }
$$

A soma da linha na matriz de transição é igual a 1 e, portanto, por notação,

$$
\sum_{j=1}^{n} P_{i j}=1 \text {. }
$$


Se $n_{i j}$ é o número de transições do estado $i$ para o estado $j$ observadas na sequência de dados, a estimativa de probabilidades de transição é dada por

$$
\hat{p}_{i j}=\frac{n_{i j}}{\sum_{i, j=1}^{n} n_{i j}} .
$$

Se $Q_{k}$ e $Q_{k+1}$, são os vetores de probabilidade observados após $k$ e $(k+1)$ passos respectivamente, a transição de $Q_{k}$ para $Q_{k+1}$ é expressa como

$$
Q_{k+1}=Q_{k} P .
$$

De acordo com as propriedades ergódicas das cadeias de Markov, o vetor de probabilidade $Q$, obtido após um longo período de tempo, torna-se independente do estado inicial, e a relação (6) pode ser escrita como

$$
Q P=Q .
$$

As soluções para esta equação geram as probabilidades limites dos estados. Estas probabilidades também podem ser estimadas a partir de dados, calculando a probabilidade empírica correspondente $P_{i}=N_{i} / M$ em que $N_{i}$ é o número de ocorrências do estado $i(i=1, \ldots, n)$ em dados observados, e $M$ é o número total das observações (Ettoumi, Sauvageot, \& Adane, 2003).

A perda de informação causada pela classificação de dados de vento em $n$ estados é dada por (Ettoumi, Sauvageot, \& Adane, 2003).

$$
L=1-H
$$

Em que $H$ é a entropia Shannon normalizada calculada utilizando as probabilidades empíricas

$$
H=\frac{1}{\log _{2}\left(\frac{1}{n}\right)} \sum_{i=1}^{n} P_{i} \log _{2} P_{i} .
$$

\section{Resultados e Discussão}

Na Figura 2, tem-se a série temporal da dispersão da velocidade do vento $(\mathrm{em} \mathrm{m} / \mathrm{s})$ registrados em cada hora a 10 metros de altura durante o período 01/01/2015-2018 na estação meteorológica de Petrolina - PE. Cadeias de Markov de primeira ordem com dois estados aplicou-se na série completa (01/01/2010-31/07/2020).

Figura 2. Série temporal horária de velocidade de vento em Petrolina.

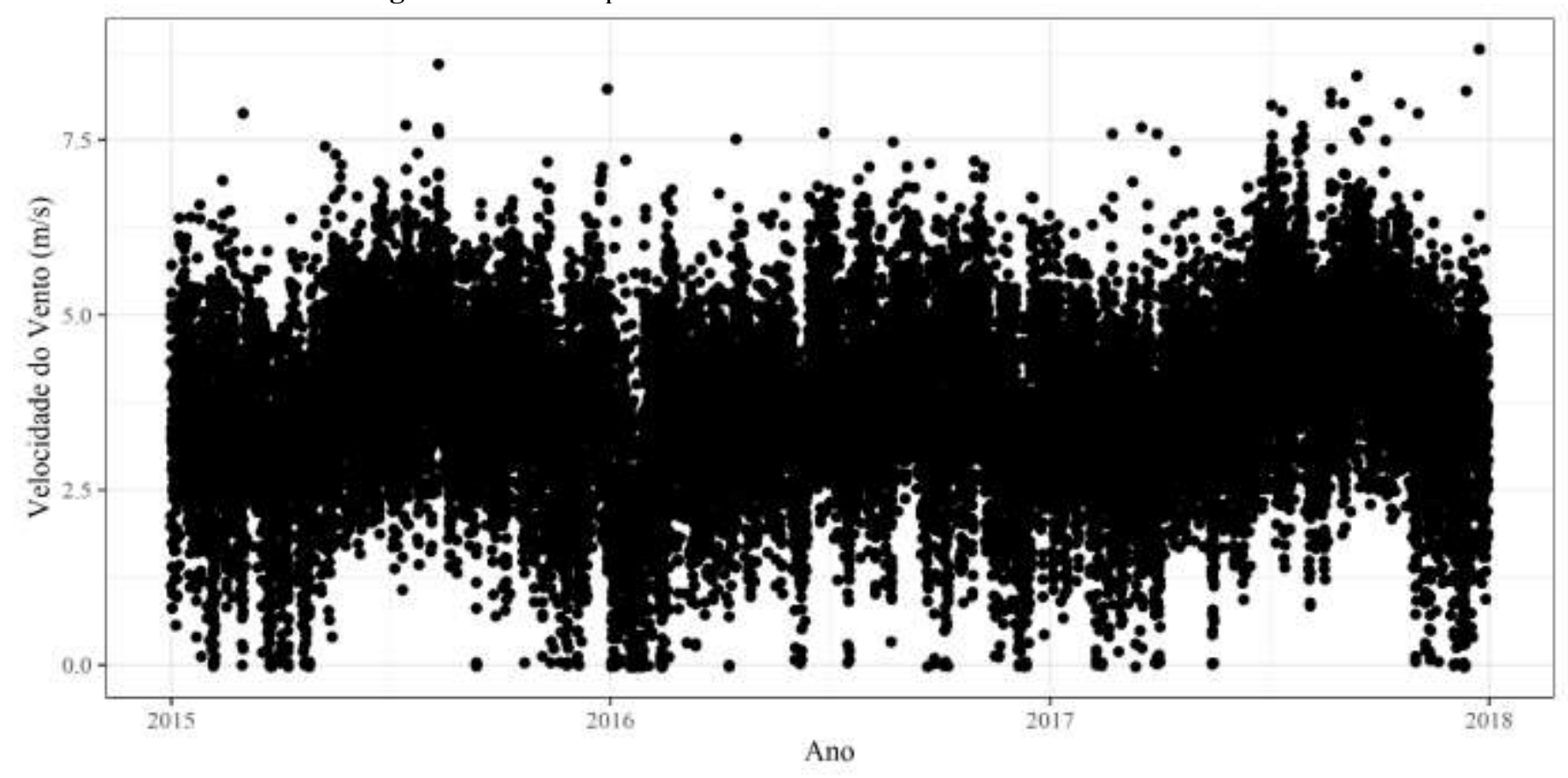

Fonte: Autores (2021). 
Os gráficos de $P_{11}, P_{12}, P_{21} \mathbf{e} P_{22}$, apresentados na Figura 3, ilustram o cálculo das probabilidades de transição de Markov para a velocidade do vento ao longo do ano. Os resultados mostram que a probabilidade $P_{11}$ é maior do que $P_{22}$ até o final do mês de abril e entre outubro e dezembro, enquanto $P_{22}>P_{11}$ ocorre entre maio e outubro, o que indica potencial eólico significativo durante estes meses.

Figura 3. Probabilidades de transição $P_{11}, P_{12}, P_{21}$ e $P_{22}$ para os dados mensais de velocidade do vento em Petrolina.

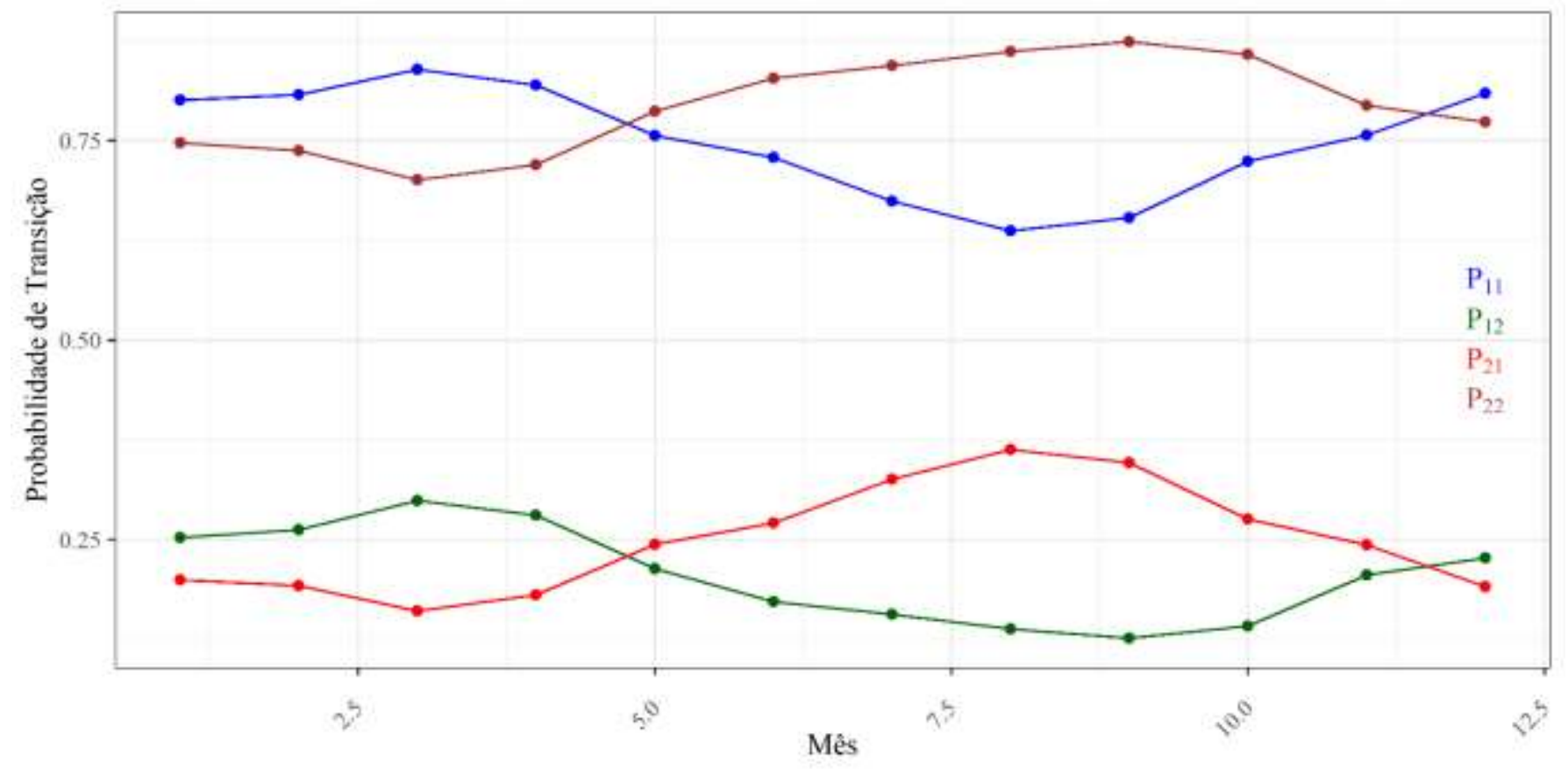

Fonte: Autores (2021).

Para cada mês do ano, a probabilidade limite de cada estado de velocidade do vento $\left(Q_{1}\right.$ e $\left.Q_{2}\right)$ foi comparada com a probabilidade empírica correspondente $\left(P_{1}\right.$ e $\left.P_{2}\right)$. Os resultados são mostrados na Figura 4. Nota-se que ambos os tipos de probabilidade são idênticos para cada um dos dois estados nos meses de janeiro, fevereiro, agosto, setembro, outubro, novembro e dezembro. Este resultado mostra que as cadeias de Markov de dois estados de primeira ordem ajustam-se bem às variações da velocidade do vento a cada uma hora, nestes meses. Em outros meses, um modelo com mais estados e/ou cadeias de Markov de maior ordem é mais indicado. 
Figura 4. Probabilidades limite $Q$ e probabilidade empírica $P$ para dois estados $(V<3,5 m / s$ e $V \geq 3,5 m / s)$ para os dados mensais de velocidade do vento em Petrolina.

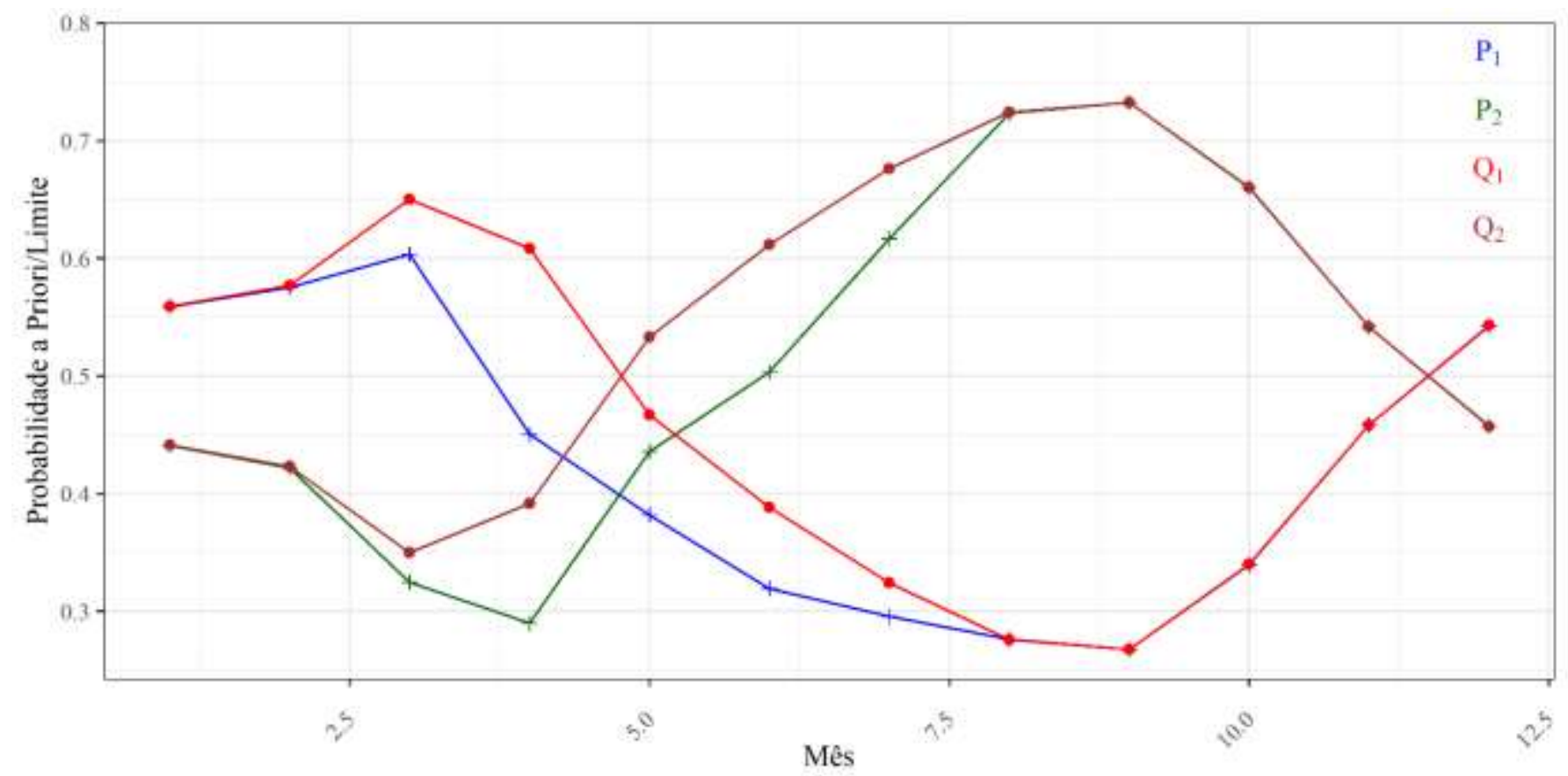

Fonte: Autores (2021).

A Figura 5 apresenta a entropia Shannon calculada para cada mês do ano usando a distribuição empírica. A entropia é mínima em outubro, quando é igual a 72\%, e máxima em maio, quando chega a aproximadamente 77,5\%. Logo, a perda de informação é relativamente alta (entre 22,5\% e 28\%), indicando que a cadeias de Markov de primeira ordem com dois estados não modela com alta precisão as flutuações da velocidade do vento em Petrolina - PE.

Figura 5. Variação anual de Entropia normalizada para os dados de velocidade do vento em Petrolina.

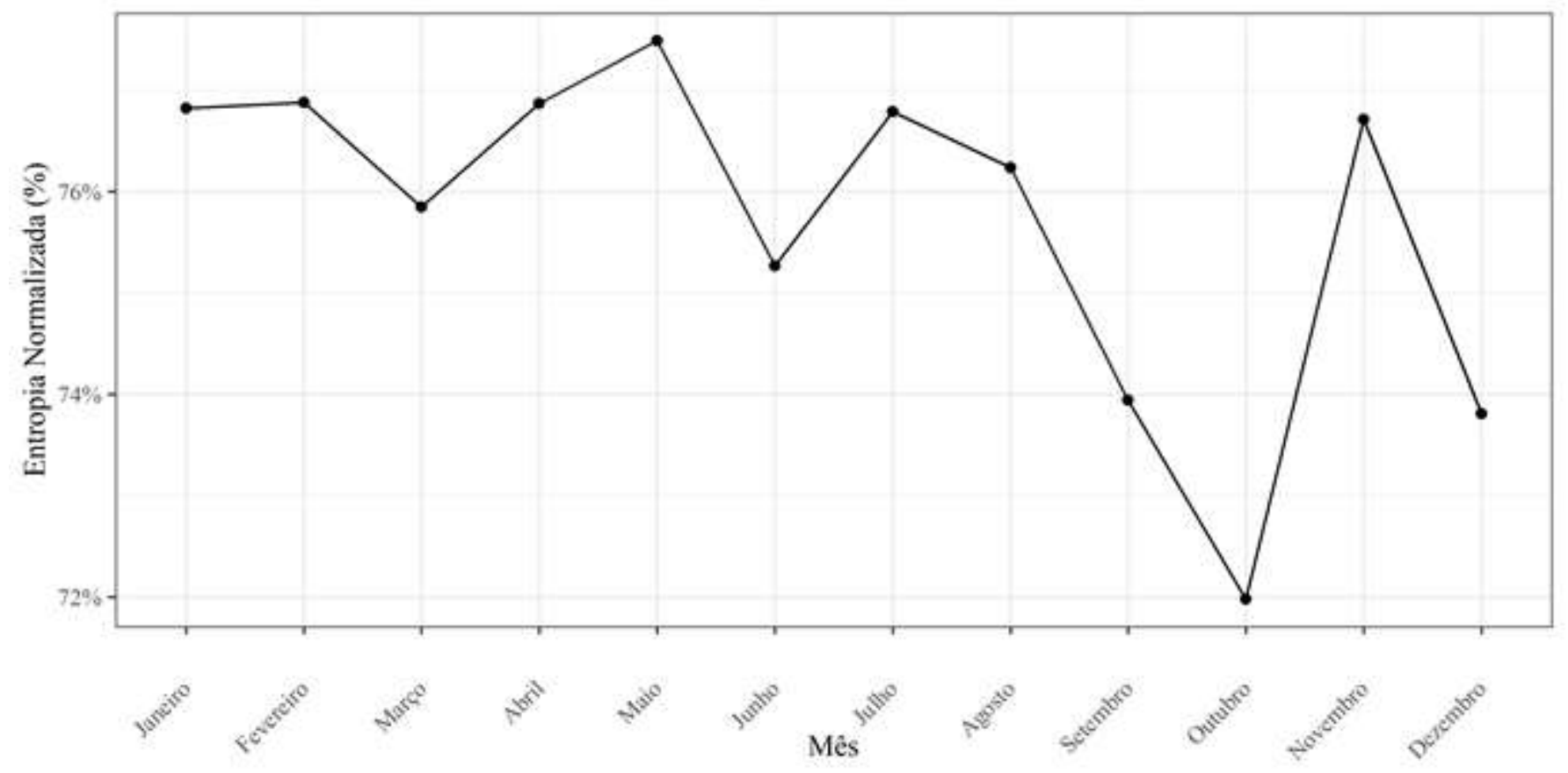

Fonte: Autores (2021). 


\section{Conclusão}

Os resultados contribuem para um melhor entendimento sobre a modelagem probabilística da dinâmica da velocidade do vento na estação de Petrolina - PE, Brasil, fornecendo informações importantes para o desenvolvimento de modelos teóricos e computacionais para o estudo deste fenômeno. Os dados de velocidade do vento de janeiro, fevereiro e de agosto a dezembro foram bem ajustados por cadeias de Markov de primeira ordem com dois estados. Isso foi verificado, fazendo um estudo comparativo entre a probabilidade empírica e a probabilidade limite de cada estado. Por outro lado, a precisão da modelagem não foi alta quando considerada a variação anual de entropia normalizada utilizando apenas dois estados.

Para trabalhos futuros, sugere-se aplicar modelos de cadeia de Markov com maior ordem e com mais estados, para investigar alterações na precisão da modelagem para este tipo de fenômeno, como também trabalhar com a variável direção do vento e com séries artificiais.

\section{Agradecimentos}

À Universidade Federal Rural de Pernambuco (UFRPE) e ao Programa de Pós-Graduação em Biometria e Estatística Aplicada (PPGBEA). A presente pesquisa foi realizada com o apoio da Coordenação de Aperfeiçoamento de Pessoal de Nível Superior - Brasil (CAPES) - Código de Financiamento 001, e com o apoio do Conselho Nacional de Desenvolvimento Científico e Tecnológico (CNPq).

\section{Referências}

ABEEólica. (2018). Annual wind energy report. http://abeeolica.org.br/wp-content/uploads/2019/06/Boletim-Anual_2018_Inglês.pdf

Bagal, H. A., Soltanabad, Y. N., Dadjuo, M., Wakil, K., \& Ghadimi, N. (2018). Risk-assessment of photovoltaic-wind-battery-grid based large industrial consumer using information gap decision theory. Solar Energy, 169, 343-352.doi.org/10.1016/j.solener.2018.05.003

Balzter, H. (2000). Markov chain models for vegetation dynamics. Ecological modelling, 126(2-3), 139-154. doi.org/10.1016/S0304-3800(00)00262-3

Carta, J. A., Ramirez, P., \& Velazquez, S. (2009). A review of wind speed probability distributions used in wind energy analysis: Case studies in the Canary Islands. Renewable and sustainable energy reviews, 13(5), 933-955. doi.org/10.1016/j.rser.2008.05.005

Chitsaz, H., Amjady, N., \& Zareipour, H. (2015). Wind power forecast using wavelet neural network trained by improved Clonal selection algorithm. Energy conversion and Management, 89, 588-598.doi.org/10.1016/j.enconman.2014.10.001

de Figueirêdo, B. C. L., Moreira, G. R., Stosic, B., \& Stosic, T. (2014). Multifractal analysis of hourly wind speed records in Petrolina, Northeast Brazil. Revista Brasileira de Biometria, 32(4), 599-608. Recuperado em 8 de abril de 2021, de http://jaguar.fcav.unesp.br/RME/fasciculos/v32/v32_n4/A9_Barbara.pdf

Ettoumi, F. Y., Sauvageot, H., \& Adane, A. E. H. (2003). Statistical bivariate modelling of wind using first-order Markov chain and Weibull distribution. Renewable energy, 28(11), 1787-1802.doi.org/10.1016/S0960-1481(03)00019-3

Global Wind Energy Council. (2019). Global wind report: Annual market update. Recuperado em 3 de junho de 2020, de https://gwec.net/global-wind-report2019

Global Wind Energy Council. (2021). Global Wind Report, 2021. Recuperado em 8 de abril de 2021, de https://gwec.net/global-wind-report-2021/

Wickham, H. ggplot2: Elegant Graphics for Data Analysis. Springer-Verlag New York. 2016.

INMET - Instituto Nacional de Meteorologia, https://portal.inmet.gov.br/dadoshistoricos

Jiao, R., Huang, X., Ma, X., Han, L., \& Tian, W. (2018). A model combining stacked auto encoder and back propagation algorithm for short-term wind power forecasting. Ieee Access, 6, 17851-17858. 10.1109/ACCESS.2018.2818108.

Jung, C., \& Schindler, D. (2019). Wind speed distribution selection-A review of recent development and progress. Renewable and Sustainable Energy Reviews, 114, 109290.doi.org/10.1016/j.rser.2019.109290

Kaygusuz, K. (2012). Energy for sustainable development: A case of developing countries. Renewable and Sustainable Energy Reviews, 16(2), 11161126.doi.org/10.1016/j.rser.2011.11.013

Masseran, N. (2015). Markov chain model for the stochastic behaviors of wind-direction data. Energy conversion and management, 92, 266274.doi.org/10.1016/j.enconman.2014.12.045

Poggi, P., Muselli, M., Notton, G., Cristofari, C., \& Louche, A. (2003). Forecasting and simulating wind speed in Corsica by using an autoregressive model. Energy conversion and management, 44(20), 3177-3196.doi.org/10.1016/S0196-8904(03)00108-0 
Research, Society and Development, v. 10, n. 9, e3610917435, 2021

(CC BY 4.0) | ISSN 2525-3409 | DOI: http://dx.doi.org/10.33448/rsd-v10i9.17435

Ruffato-Ferreira, V., Barreto, R. C., Júnior, A. O., Silva, W. L., Viana, D. B., Nascimento, J. A. S., \& Freitas, M. A. V. (2017). A foundation for the strategic long-term planning of the renewable energy sector in Brazil: Hydroelectricity and wind energy in the face of climate change scenarios. Renewable and Sustainable Energy Reviews, 72, 1124-1137.doi.org/10.1016/j.rser.2016.10.020

Sadorsky, P. (2021). Wind energy for sustainable development: Driving factors and future outlook. Journal of Cleaner Production, 289, 125779.doi.org/10.1016/j.jclepro.2020.125779

Sarmiento, C., Valencia, C., \& Akhavan-Tabatabaei, R. (2018). Copula autoregressive methodology for the simulation of wind speed and direction time series. Journal of Wind Engineering and Industrial Aerodynamics, 174, 188-199. doi.org/10.1016/j.jweia.2018.01.009

Shamshad, A., Bawadi, M. A., Hussin, W. W., Majid, T. A., \& Sanusi, S. A. M. (2005). First and second order Markov chain models for synthetic generation of wind speed time series. Energy, 30(5), 693-708. doi.org/10.1016/j.energy.2004.05.026

Simas, M., \& Pacca, S. (2013). Energia eólica, geração de empregos e desenvolvimento sustentável. Estudos avançados, 27(77), 99-116. doi.org/10.1590/S010340142013000100008

Spedicato, G. A. Discrete Time Markov Chains with R. The R Journal, 2017.

Van Kooten, G. C., Duan, J., \& Lynch, R. (2016). Is there a future for nuclear power? Wind and emission reduction targets in fossil-fuel Alberta. PloS one, 11(11), e0165822. https://doi.org/10.1371/journal.pone.0165822

Xie, K., Liao, Q., Tai, H. M., \& Hu, B. (2017). Non-homogeneous Markov wind speed time series model considering daily and seasonal variation characteristics. IEEE Transactions on Sustainable Energy, 8(3), 1281-1290. 10.1109/TSTE.2017.2675445 